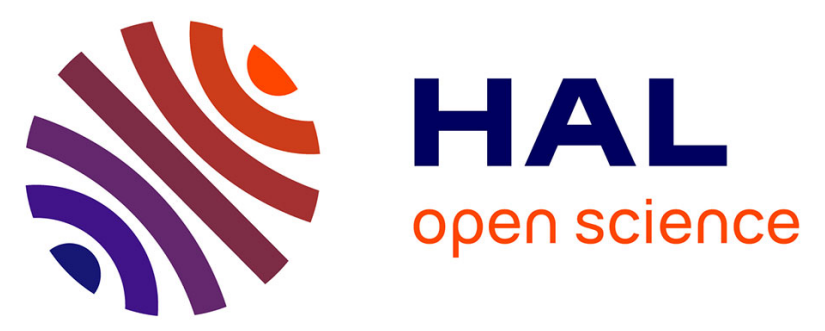

\title{
Lessons learned from nuclear decay data measurements in the european metrology research programme 'MetroFission' (June 2013)
}

S. Pomme, Martin Loidl, E. García-Torano, M. Marouli, Cindy Le Bret, M.T.

Crespo, J. Paepen, Xavier Mougeot, V. Jobbágy, Matias Rodrigues, et al.

\section{To cite this version:}

S. Pomme, Martin Loidl, E. García-Torano, M. Marouli, Cindy Le Bret, et al.. Lessons learned from nuclear decay data measurements in the european metrology research programme 'MetroFission' (June 2013). 3rd International Conference on Advancements in Nuclear Instrumentation, Measurement Methods and their Applications (ANIMMA), Jun 2013, Marseille, France. pp.6727934, 10.1109/ANIMMA.2013.6727934 . cea-01822351

\section{HAL Id: cea-01822351 https://hal-cea.archives-ouvertes.fr/cea-01822351}

Submitted on 11 Feb 2020

HAL is a multi-disciplinary open access archive for the deposit and dissemination of scientific research documents, whether they are published or not. The documents may come from teaching and research institutions in France or abroad, or from public or private research centers.
L'archive ouverte pluridisciplinaire HAL, est destinée au dépôt et à la diffusion de documents scientifiques de niveau recherche, publiés ou non, émanant des établissements d'enseignement et de recherche français ou étrangers, des laboratoires publics ou privés. 


\title{
Lessons Learned From Nuclear Decay Data Measurements in the European Metrology Research Programme 'MetroFission' (June 2013)
}

\author{
S. Pommé, M. Loidl, E. García-Toraño, M. Marouli, C. Le-Bret, M.T. Crespo, J. Paepen, X. Mougeot, \\ V. Jobbágy, M. Rodrigues, R. Van Ammel, H. Stroh, A. Luca
}

\begin{abstract}
Nuclear decay data measurements were performed in the frame of a EURAMET project. Two specific goals were addressed: (1) High-precision measurements of ${ }^{238} U$ alphaparticle emission probabilities and (2) experimental characterization of the shape of beta spectra using cryogenic detectors. In the paper, specific lessons learned from the metrological work are highlighted. This includes effects of source preparation on the quality of alpha and beta spectrometry, the influence of exchange effects on beta spectra and the use of a magnet system to eliminate coincidence effects by conversion electrons in alpha-particle spectra.
\end{abstract}

Index Terms-alpha spectrometry, beta spectrometry, cryogenic detector, source preparation, exchange effect, coincidence, magnet

\section{INTRODUCTION}

$\mathrm{F}$ OLLOWING the EURAMET EMRP Energy call of 2009, the research project "Metrology for new generation nuclear power plants" or "MetroFission" was funded for a 3 years period. The project started in September, 2010 and was run by a European consortium of 12 partners [1]. It aimed at answering several metrological problems related to the development of the proposed Generation IV nuclear reactors design. Specific aspects related to the nuclear decay data improvement were treated by the Work Package 5: high precision measurements of ${ }^{238} \mathrm{U}$ alpha-particle emission probabilities and experimental characterization of the beta

7 May 2013 "The research leading to these results has received funding from the European Union on the basis of Decision No 912/2009/EC".

S. Pommé, M. Marouli, J. Paepen, V. Jobbágy, R. Van Ammel and H. Stroh are with the Joint Research Centre of the European Commission, Institute for Reference Materials and Measurements, B-2440 Geel, Belgium (e-mail: stefaan.pomme@ec.europa.eu).

M. Loidl, C. Le-Bret, X. Mougeot and M. Rodrigues are with the LNE Laboratoire National Henri Becquerel, CEA Saclay, DRT/LIST/DM2I, 91191 Gif-sur-Yvette, France (e-mail: martin.loidl@cea.fr).

E. García-Toraño and T. Crespo are with the Laboratorio de Metrología de Radiaciones Ionizantes, CIEMAT, 28040 Madrid, Spain (e-mail: e.garciatorano@ciemat.es).

A. Luca is with the IFIN-HH, DRMR/Radionuclide Metrology Laboratory, Magurele, Romania (e-mail: aluca@nipne.ro) spectral shape using cryogenic detectors. These new measurements respond to user requests identified by the IAEA, according to the CRP F42006 (Updated decay data library for Actinides) [2] and two consultants meetings on the calculation of the decay heat induced by nuclear fission. The results will be used in the future to solve some present inconsistencies and perform better nuclear structure and decay data evaluations by the international networks of evaluators. To this aim, an additional work package foresaw in the organization of an international workshop in 2013, open to all the nuclear data evaluators and users, scientists, engineers, professors and students. In the paper some specific lessons learned from the metrological work are highlighted.

Beta spectrometry by means of a cryogenic detector is a novel technique that needed quite some testing to become operable. One of the issues was the dependence of the spectral distribution on the source production method. Tests with drop deposited sources were unsatisfactory: the measured spectra deviated from theory and from one another. It was found that the spectra resulting from electrodeposited sources were reproducible and closer to the theoretical spectrum. Still, there was a deviation from a standard calculation of the spectrum of an allowed beta transition, which could be solved by including the atomic exchange effect into the model. These measurements are a clear proof of the presence of exchange effects in beta decay and emphasize the need to account for them.

In alpha-particle spectrometry one was faced with peculiar problems due to the extremely long half-life of ${ }^{238} \mathrm{U}$. The low specific activity of the enriched uranium material required the use of relatively high amounts in the source, which was contradictory to the requirement of having a thin layer for optimum energy resolution. Quite some research went into the production of sources by electrodeposition that could optimize between both requirements. An additional problem was the relatively short distance between source and detector to collect sufficient events for statistical accuracy. As a consequence, a significant amount of conversion electrons were detected coincidently with the alpha particles, leading to a distortion of the spectrum and wrong assignment of emission probabilities. In spite of mathematical corrections, measurements in 
different geometries lead to different results. This could only be solved by the design and implementation of a new magnet system to eliminate conversion electrons.

\section{ALPHA SPECTROMETRY}

The high-resolution alpha spectrometers at CIEMAT and IRMM [3] are dedicated to the improvement of our knowledge of nuclear decay data, such as alpha emission energies and probabilities. Ion-implanted silicon detectors with a relatively low active area of $50 \mathrm{~mm}^{2}$ are typically used because of their superior energy resolution. Sources are preferably made of highly enriched material of the radionuclide of interest, to avoid interferences by the activity of other radionuclides present as impurities in the material. For optimum energy resolution of the alpha spectra, normally thin point-like sources are prepared by techniques that allow for a homogenous deposition with low self-absorption, such as vacuum evaporation or electrodeposition. The sources are preferably measured at a sufficient distance from the detector, to ensure that a low solid angle is covered. This has a beneficial effect on the energy resolution and reduces the problem of simultaneous detection of conversion electrons with the alpha particles, which would distort the energy spectrum and would lead to errors in the derived alpha emission probabilities. Spectrum deconvolution software is then used to fit the spectra and obtain the area underneath the individual peaks.

The problem with extremely long-lived alpha emitters like ${ }^{238} \mathrm{U}$ is their low specific activity, which makes it impossible to use the normal procedure described above. One cannot use a thin point source as it would not contain enough activity. Therefore bigger sources are needed, but still sufficiently thin to maintain a reasonably good energy resolution. One cannot measure too far away from the detector for the same reason of obtaining sufficient statistical accuracy. Having compromised a bit on energy resolution through the size and thickness of the source, one can also allow using a slightly bigger detector of $150 \mathrm{~mm}^{2}$ or more, without too much extra loss of resolving power. However, the increase of solid angle covered by the detector entails a significant impact of coincidence effects between alpha particles and conversion electrons. One can calculate their abundance and try to correct for them mathematically. Alternatively, the conversion electrons can be deflected by a magnet system. However, the existing magnet systems were designed for point-like sources only.

\section{A. Source Preparation}

The isotopic abundance of ${ }^{238} \mathrm{U}$ in the enriched uranium material was $99.99922 \%$ (mass percent) and $99.9815 \%$ in activity (with $0.01818 \%$ of ${ }^{234} \mathrm{U}$ activity). Electrodeposition was preferred over vacuum evaporation as source preparation technique because of its higher yield. However, many trial tests had to be performed to find the ideal conditions, which involves many free parameters such as source diameter, thickness, electrolyte (composition, $\mathrm{pH}$, temperature), anode (material, rotation and geometry), purity of materials, current homogeneity, substrate surface roughness, calcination of source, etc. The different parameters tested at IRMM and CIEMAT have been described in detail by Jobbágy et al [4].

Three different types of electrolytes were tested. The first one was an organic (iso-propanol) electrolyte, while the other two were inorganic electrolytes: $\mathrm{H}_{2} \mathrm{SO}_{4} /\left(\mathrm{NH}_{4}\right)_{2} \mathrm{SO}_{4}$ and saturated $\mathrm{NH}_{4} \mathrm{NO}_{3}$. The homogeneity of the uranium sources was investigated by autoradiography. Energy resolution, lowenergy tailing and deposited activity were checked by high resolution alpha-particle spectrometry, which was quite time consuming.

The best compromise between counting statistics and good energy resolution was achieved with sources produced in saturated $\mathrm{NH}_{4} \mathrm{NO}_{3}$, using a modified version of the method introduced by Dos Santos et al. [5][6], and limiting the surface density to $26 \mu \mathrm{g} \mathrm{cm}^{-2}$. The sources had about $1 \mathrm{~Bq}$ activity. The peak-to-valley ratio between the main peaks was about 60 and the resolution between 15 and $18 \mathrm{keV}$.

At CIEMAT, the cell consisted of a polypropylene tube, the electrodes were a mirror-polished stainless steel cathode disc and a 1-mm-diameter platinum anode wire bent into a spiral, the electrolyte was $8 \mathrm{~mL}$ saturated ammonium nitrate solution, $2 \mathrm{~mL}$ ultrapure water, without $\mathrm{pH}$ adjustment, the current density was $0.5 \mathrm{~A} / \mathrm{cm}^{2}$ with $10 \mathrm{~mm}$ distance between the electrodes, the cathode had a $22 \mathrm{~mm}$ active diameter and the electrodeposition time was $120 \mathrm{~min}$. The cell was immersed into a water bath for refrigeration and in some occasions ultrasonic agitation was applied. Comparable conditions were used at IRMM.

\section{B. Measurements}

In spite of the larger sources and detectors used, the count rate was still low and measurements had to be continued for 12 years on three set-ups to obtain enough information to lower the uncertainty of the decay data by one order of magnitude. This required thermal stabilization of the whole set-up, by means of a climatized laboratory and a continuous flow of water from a thermostatic bath around the housing of the detector and pre-amplifier. Spectra were taken at regular intervals and energy drift was compensated off-line by spectral shift using dedicated software based on a Stieltjes integral [7] or Fourier analysis [8].

Spectra were taken at different solid angles and analyzed by spectrum deconvolution. The obtained relative peak areas were mathematically corrected for the summing-out and summing-in effects that theoretically could be expected from coincidence effects. However, it turned out that the mathematics could not completely compensate for the spectral distortions caused by coincidental detections of alpha particles and electrons. The derived emission probabilities still varied significantly as a function of the solid angle subtended by the detector. This puts in question the attainable accuracy of alpha spectrometry, not only for ${ }^{238} \mathrm{U}$ but also for other alpha emitters measured in close range and susceptible to conversion electron emission. 


\section{Magnet System}

During the course of the project, Paepen et al. [9] designed and built a new magnet system that could host sources with a diameter up to $30 \mathrm{~mm}$. The dimensions of the magnets were limited to allow for easy access of the magnetic system into the source chamber. The magnetic field strength was optimized so that conversion electrons below a certain energy would be deflected from the detector or caught in the field between the two opposing magnets. Two yokes were built which allow varying the distance between the magnets, with the closer geometry creating a stronger field and enhanced capacity to remove electrons of higher energy. For ${ }^{238} \mathrm{U}$, an aperture of $36 \mathrm{~mm}$ was chosen, which is effective up to an electron energy of at least $80 \mathrm{keV}$. At $20 \mathrm{~mm}$ distance, the effectiveness extends to $300 \mathrm{keV}$.

Spectra were taken with the magnet (see for example Fig. 1) at different solid angles and this time the resulting alpha emission probabilities were almost invariable. As a result, a series of measurements could be performed at even closer range to significantly increase the count rate. The result was hardly influenced by these low distances, which would be unthinkable when measuring without magnet system. As a result, we obtained more accurate and statistically more precise values that agreed well with the extrapolation value towards zero solid angle of measurements performed without magnet [10].

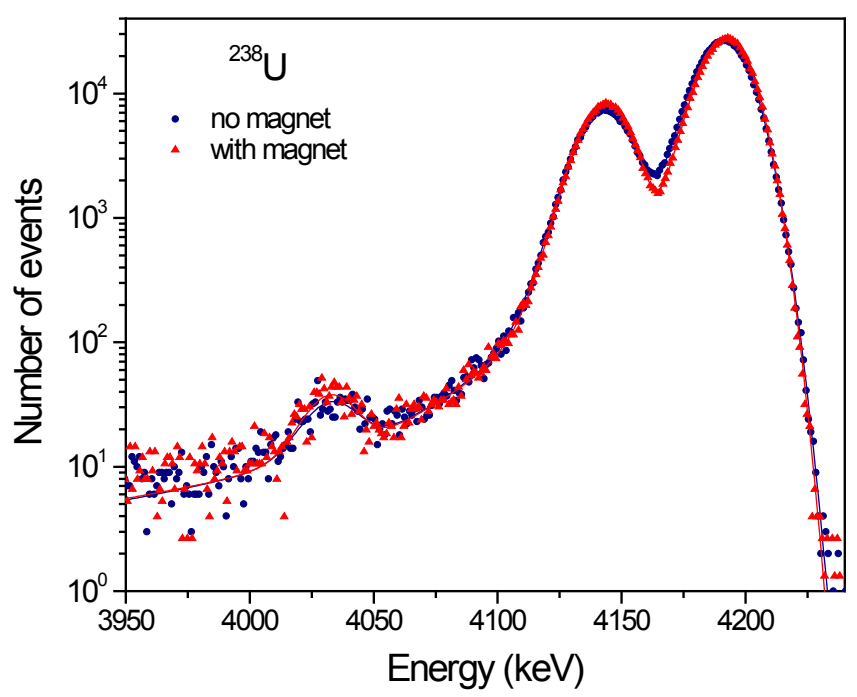

Fig. 1. Alpha spectra of ${ }^{238} \mathrm{U}$ measured with and without magnet system. The spectrum with magnet is less distorted, shows a better energy resolution and its relative peak areas are not affected by summing effects with conversion electrons.

\section{BETA SPECTROMETRY}

Cryogenic detectors, more specifically metallic magnetic calorimeters, are used for beta spectrometry firstly because of their high energy resolution and low energy threshold [11], and secondly because it is straightforward to realize a solid angle of $4 \pi \mathrm{sr}$ and high detection efficiency by enclosing the beta emitter in the detector absorber. This helps also avoiding backscattering at the detector surface. The detector operates at a temperature between 10 and $20 \mathrm{mK}$ and the energy of each beta particle is measured as a temperature rise via a paramagnetic $\mathrm{Au}: \mathrm{Er}$ thermometer and a SQUID magnetometer. The temperature equilibrium is restored via a thermal link between the detector and a thermal bath. The relatively long thermal time constant, of the order of a millisecond, is compatible with the low activity sources (a few $\mathrm{Bq}$ ) used in this work.

\section{A. ${ }^{63} \mathrm{Ni}$ : Impact of the Source Preparation}

It was decided to measure as a first step the spectrum of the pure beta emitter ${ }^{63} \mathrm{Ni}$ (half-life 98.7 (24) years, endpoint energy $66.980(15) \mathrm{keV}$ [12]) which decays via an allowed transition. Spectra resulting from allowed transitions can be calculated quite reliably, yielding a reference spectrum to compare with the experimental spectrum in order to validate the detection method.

Several ${ }^{63} \mathrm{Ni}$ sources were prepared by drop deposition of a $\mathrm{NiCl}_{2}$ solution on thin gold foils and dried at room temperature. Each source was covered with a second gold foil and the pairs of gold foils were diffusion welded, thus hermetically enclosing the sources. The sandwich gold foil source - gold foil constitutes the detector absorber. The thickness of the gold foils $(\geq 10 \mu \mathrm{m})$ was determined by Monte Carlo simulations so as to fully stop all electrons up to the maximum energy of the spectrum. The simulations also confirmed that the energy loss by the escape of Bremsstrahlung photons is negligible. The detection efficiency is $100 \%$ above the energy threshold of $200 \mathrm{eV}$.

The detector linearity was checked and confirmed with $\mathrm{X}$ and gamma ray lines from ${ }^{55} \mathrm{Fe},{ }^{109} \mathrm{Cd}$ and ${ }^{241} \mathrm{Am}$ sources: the residuals were less than $0.1 \%$ in the energy range $6-88 \mathrm{keV}$. During the measurements of the beta spectrum, only the $59.5 \mathrm{keV}$ line of an ${ }^{241} \mathrm{Am}$ source was used for energy calibration.

The measured spectra differed from one source to another and all of them diverged from the theoretical spectrum, in particular at very low energy [13]. The spectral distortion was attributed to the fact that part of the electron energy is deposited in the salt forming the ${ }^{63} \mathrm{Ni}$ source. A non-negligible fraction of this energy may be expended in the creation of metastable states, like electron-hole pairs, that do not produce heat on a sufficiently short time scale. This part of the energy would be lost for detection. However, there is no proof for this hypothesis.

Upon the difficulties encountered with dried drop-deposited sources, several ${ }^{63} \mathrm{Ni}$ sources were made by electroplating on gold and silver foils, yielding metallic Ni deposits. Energy loss by creation of electron-hole pairs can be excluded in this case. The spectra measured with two electroplated sources, one in a gold absorber and one in a silver absorber, had an identical shape. But there remained a discrepancy with respect to the theoretical spectrum calculated according to a standard approach including the usual corrections for screening, radiative and nuclear size effects. 


\section{B. Exchange Effect}

This discrepancy could be resolved by including the atomic exchange effect into the theoretical model of the beta decay. The exchange effect corresponds to the case where a beta electron is not directly emitted into the continuum but into a bound orbital of the daughter atom, accompanied by the simultaneous emission of a bound electron. It leads to an enhancement of the beta emission intensity, in particular at low energy. It was first discussed in [14] and quantitatively evaluated for some beta decaying nuclides in [15]. It was implemented for allowed transitions in the code BetaShape developed at LNHB [16].

None of the spectra measured with drop-deposited sources agrees perfectly with any of the theoretical spectra, but the discrepancy is much smaller when taking into account the exchange effect than without the exchange effect. The spectra obtained with electroplated sources are in excellent agreement with the theoretical shape if the exchange effect is included. Fig. 2 shows the spectrum recorded with the gold absorber together with theoretical spectra calculated with and without exchange effect. This figure demonstrates the strong impact of the exchange effect in the decay of ${ }^{63} \mathrm{Ni}$ : at the energy threshold of $200 \mathrm{eV}$ the enhancement of the emission probability is as high as $23 \%$.

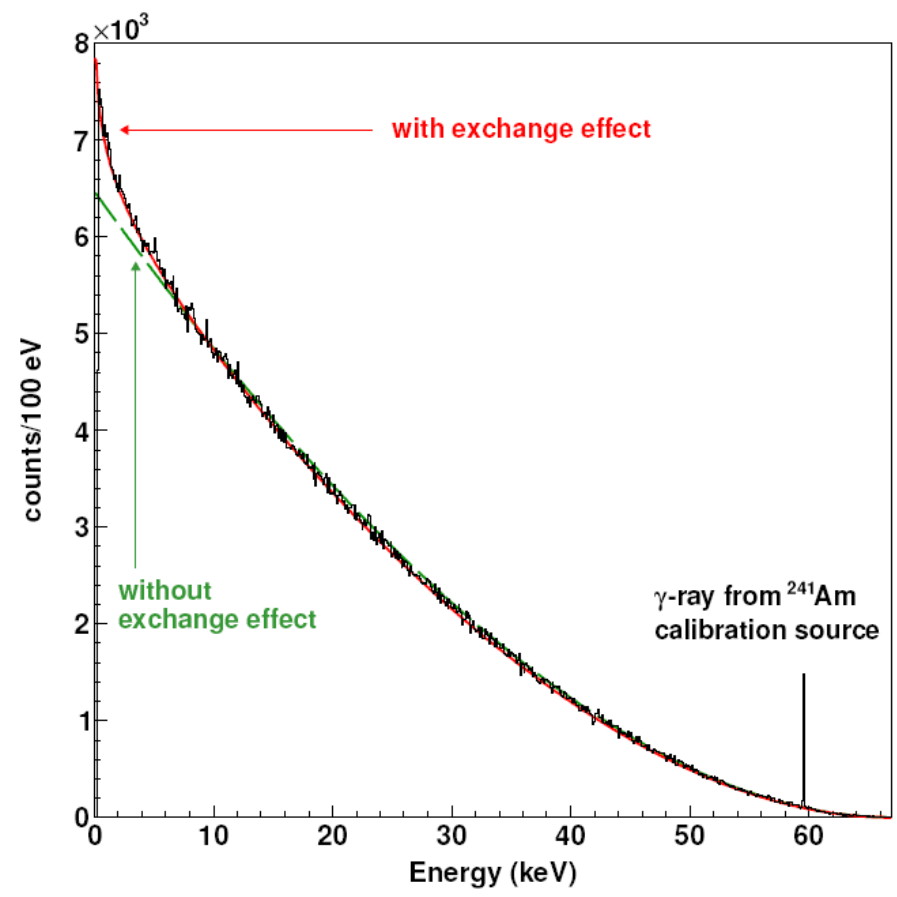

Fig. 2. Beta spectrum of an electroplated ${ }^{63} \mathrm{Ni}$ source measured with a metallic magnetic calorimeter ("rugged" black line), compared with theoretical spectra with (smooth solid line) and without (dashed line) exchange effect.

The exchange effect has also an influence on the mean energy of the beta emissions. For ${ }^{63} \mathrm{Ni}$ it is lower by $2.3 \%$ with respect to a calculation without exchange effect. In other words, not taking into account the exchange effect leads to an overestimation of the mean energy. Hence the exchange effect should be taken into account in the calculation of beta spectra, although its contribution is less pronounced for high-energy beta emitters or low-mass nuclei. The standard approach of calculating beta spectra is incomplete, even for allowed transitions.

This experimental work is the most convincing evidence for the exchange effect in beta decay. Two previous experiments had already confirmed its existence, first qualitatively [17] and later also quantitatively [18], but with a much higher threshold at several $\mathrm{keV}$. The present work also demonstrates that the computational treatment of the exchange effect is correct even at very low energy, where it is the most significant and where wrong assumptions in the calculation would be much more evident than at several $\mathrm{keV}$.

\section{C. ${ }^{241}$ Pu: Forbidden Transition}

For the first measurement of a beta spectrum from a forbidden transition, the pure beta emitter ${ }^{241} \mathrm{Pu}$ was chosen. It decays via a first forbidden, non-unique transition, its half-life is 14.33 (4) years and the endpoint energy is $20.8(2) \mathrm{keV}$ [19]. The source was prepared by drop deposition of $\mathrm{Pu}$ solution and subsequent drying.

The spectrum measured with this source was compared with theoretical spectra calculated with and without exchange effect. Again, the agreement between experiment and theory is much better when the exchange effect is taken into account. The spectrum was calculated as an allowed transition, which is of course questionable for a non-unique forbidden transition. However, first forbidden non-unique transitions which fulfill the condition $2 \xi=\alpha Z / R \gg E_{0}$ can be treated as allowed ones [20], with $\alpha$ the fine structure constant, $R$ the radius of the daughter nucleus, $Z$ its nuclear charge, and $E_{0}$ the maximum energy of the transition. This approximation, called the $\xi$ approximation, means that the Coulomb energy of the beta electron at the nuclear radius must be large compared to the total decay energy $Q_{\beta}$.. ${ }^{241} \mathrm{Pu}$ fulfills very well this condition, because of its low $Q_{\beta-}=20.8 \mathrm{keV}$ compared to $2 \xi \approx$ $19.8 \mathrm{MeV}$.

A small discrepancy remaining between the experimental and the theoretical spectrum taking account of the exchange effect is most likely due to the use of a drop-deposited source. Electroplating of metallic plutonium is not possible. A source prepared by electroless plating shall be measured shortly. An excellent alternative way of source preparation would be implantation of the Pu nuclei directly into the material of the detector absorber. In this case any influence of the source on the measured spectrum can be safely excluded.

\section{CONCLUSION}

Source preparation by electrodeposition has been optimized for uranium sources to yield good energy resolution for alphaparticle spectrometry. The thickness of alpha-emitting sources is preferably kept below $26 \mu \mathrm{g} \mathrm{cm}^{-2}$ to retain an energy resolution of about $15 \mathrm{keV}$. Mathematical corrections for coincidence effects with conversion electrons do not adequately compensate for the spectral distortion. The use of a magnet system eliminates conversion electrons and allows to determine alpha emission probabilities with better accuracy and statistical precision.

The accuracy of beta spectra measured with metallic magnetic calorimeters depends crucially on the quality of the sources. 
Much better results are obtained with electroplated sources compared to dried drop-deposited sources. Excellent sources could also be produced by direct implantation of the radioactive nuclei into the absorber material, which is a good alternative for nuclides that cannot be electroplated in metallic form, such as ${ }^{241} \mathrm{Pu}$. The measurements clearly confirm the importance of the exchange effect in the low energy part of the beta spectra. It is less pronounced for high-energy beta emitters or low-mass nuclei.

\section{REFERENCES}

[1] L. Johansson, J.-R. Filtz, P. De Felice, S. Mohamed, A. Plompen, B. Hay, A. Dinsdale, S. Pommé, P. Cassette, J. Keightley. (June 2011). Advanced Metrology for New Generation Nuclear Power Plants. in: Proceedings of IMEKO-MI2011, Metrological Infrastructure, Environmental and Energy Measurement, Mediterranean Industry of the Sun, Boršić, M. (Editor), Cavtat, Croatia, June 15-17, 2011, pp. 9-13.

[2] M. A. Kellett, "Assessment of actinide decay data evaluations: Findings of an IAEA Coordinated Research," Appl. Radiat. Isot., vol. 70, pp. 1919-1923, Sept. 2012.

[3] S. Pommé and G. Sibbens, "Alpha-particle counting and spectrometry in a primary standardisation laboratory," Acta Chim. Slov., vol. 55, pp. 111-119, March 2008.

[4] V. Jobbágy, M. T. Crespo, R. Van Ammel, M. Marouli, A. Moens, S. Pommé, E. García-Toraño, "Preparation of high-resolution ${ }^{238} \mathrm{U} \alpha$ sources by electrodeposition: a comprehensive study," J. Radioanal. Nucl. Chem., 10.1007/s10967-013-2444-8, in press.

[5] L. R. Dos Santos, M. E. Sbampato and A. M. Dos Santos. "Characterization of electrodeposited uranium films," J. Radioanal. Nucl. Chem. 261, pp. 203-209, July 2004.

[6] T. Crespo, "A review of electrodeposition methods for the preparation of alpha-radiation sources," Appl Radiat Isot., vol. 70, no. 1, pp. 210-215, Jan. 2012.

[7] S. Pommé and G. Sibbens, "A new off-line gain stabilisation method applied to alpha-particle spectrometry," in: Advanced Mathematical and Computational Tools in Metrology VI, ed. Ciarlini, P., Cox, M. G., Pavese, F., Rossi, G. B., Series on Advances in Mathematics for Applied
Sciences - Vol. 66 (World Scientific Publishing Company, 2004) pp. 327-329.

[8] P. Rubio Montero and E. García-Toraño, "Use of discrete Fourier transform to sum spectra in measurements with long counting times," Nucl. Instrum. Meth. A, vol. 577, no. 3, pp. 715-718, July 2007.

[9] J. Paepen, A. Dirican, M. Marouli, S. Pommé, R. Van Ammel and H. Stroh, "A magnet system for the suppression of conversion electrons in alpha spectrometry," Appl. Radiat. Isot., in press.

[10] S. Pommé, E. García-Toraño, M. Marouli, T. Crespo, V. Jobbágy, R. Van Ammel, J. Paepen, H. Stroh, "High-resolution alpha-particle spectrometry of ${ }^{238} \mathrm{U}$," Appl. Radiat. Isot., in press.

[11] A. Fleischmann, C. Enss and G. Seidel, "Metallic Magnetic Calorimeters", in Cryogenic Particle Detectors, Topics Appl. Phys. 99, C. Enss (Ed.), Springer Berlin/Heidelberg, 2005, pp. $151-216$.

[12] K. B. Lee, Table of Radionuclides, Monographie BIPM-5 Vol. 3, edited by M.-M. Bé et al., CEA/LIST-LNHB, BIPM, 2006, p.29.

[13] C. Le-Bret, M. Loidl, M. Rodrigues, X. Mougeot and J. Bouchard, "Study of the influence of the source quality on the determination of the shape factor of beta spectra," J. Low Temp. Phys., vol. 167, pp. 985-990, March 2012.

[14] J. N. Bahcall, "Overlap and exchange effects in beta decay", Phys. Rev., vol. 129, pp. 2683-2694, March 1963.

[15] M. R. Harston and N. C. Pyper, "Exchange effects in $\beta$ decays of manyelectron atoms", Phys. Rev. A, vol. 45, pp. 6282-6295, May 1992.

[16] X. Mougeot, M.-M. Bé, C. Bisch and M. Loidl: "Evidence for the exchange effect in the beta decay of ${ }^{241}$ Pu", Phys. Rev. A, vol. 86, pp. 042506-1 - 042506-7, Oct. 2012.

[17] M. M. Lowry, D. Deptuck and I. C. Girit, "Beta spectrum studies with cryogenic micro-calorimeters", J. Low Temp. Phys., vol. 93, pp. 239244, Nov. 1993.

[18] L. C. Angrave, N. E. Booth, R. J. Gaitskell, G. L. Salmon and M. R. Harston, "Measurement of the atomic exchange effect in nuclear $\beta$ decay", Phys. Rev. Lett., vol. 80, pp. 1610-1613, Feb.1998.

[19] V. P. Chechev and N. K. Kuzmenko, Table of Radionuclides, Monographie BIPM-5 Vol. 4, edited by M.-M. Bé et al., CEA/LISTLNHB, BIPM, 2008, p.259.

[20] H.F. Schopper, Weak interactions and nuclear beta decay, NorthHolland Publishing Company, 1966. 